\section{IN THE NEWS}

Allergen-free cats

The Californian company Allerca Inc., a division of Geneticas Life Sciences, has announced its plans to produce the world's first genetically engineered allergen-free cat, which should reach the market by 2008 . The cats will cost upwards of $\$ 3,500$ and the company's press release estimates, based on the interest shown by deposits they have already received, that annual consumer sales in the United States alone will be in excess of $\$ 1.3$ billion. Allerca aim to use RNAinterference (RNAi) technology to 'silence' the gene encoding a glycoprotein known as Fel d1, which is the most common cat allergen present in saliva and on the skin. However, Thomas Platts Mills, of the Asthma and Allergic Diseases Center at the University of Virginia, points out that "cats produce many more allergens" (New Scientist). It is also unclear whether RNAi will work in cats, and research and development will not begin until 2005

Simon Brodie, President of Allerca, is appealing to the sentiments of pet lovers, who he says will be able to "enjoy the love and companionship of a pet without the cost, inconvenience, risk and limited effectiveness of current treatments". But Maggy Jennings of the RSPCA (Royal Society for the Prevention of Cruelty to Animals, UK) has a much more practical and less costly solution: "We would urge people who are allergic to cats to consider alternative companion animals."

(Independent on Sunday)

Kirsty Minton

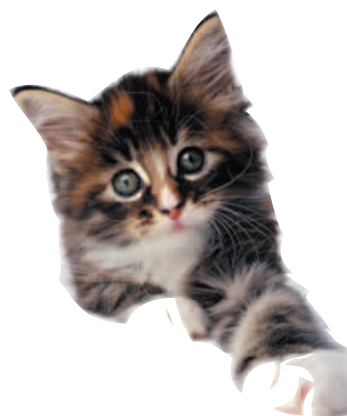

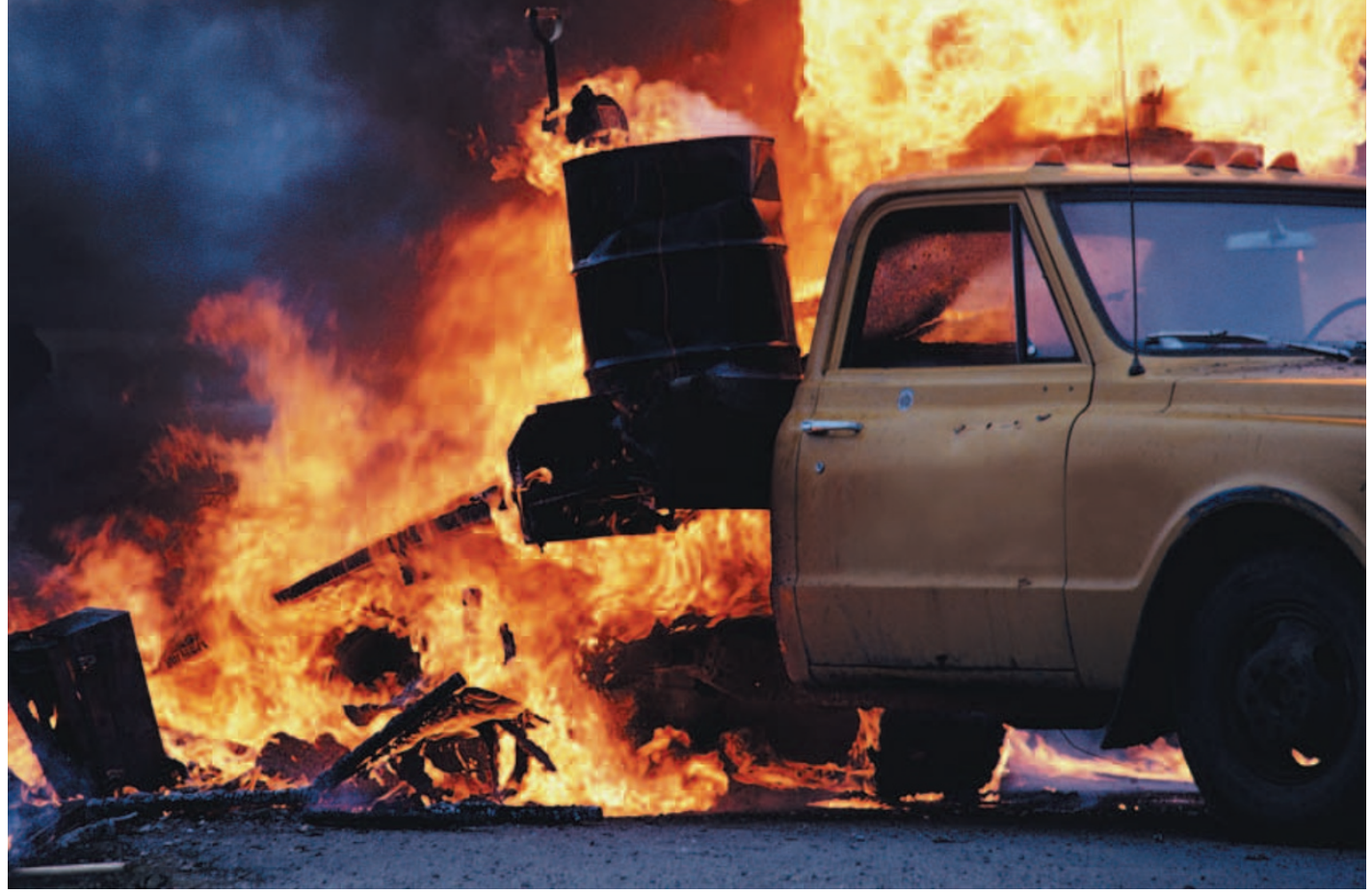

MACROPHAGES

\title{
New anti-inflammatory pathway
}

The pathological consequences of sepsis are the result of high-level release of inflammatory cytokines such as tumour-necrosis factor (TNF) and interleukin-1 $\beta$, but although neutralization of these cytokines can prevent the development of sepsis in animal models, it has little effect on established disease in patients. This led Kevin Tracey, Luis Ulloa and colleagues to identify highmobility group box 1 (HMGB1) produced by macrophages as a necessary and sufficient, late mediator of sepsis. They have now extended this work to define the first known physiological anti-inflammatory pathway to counter the effects of HMGB1, which is amenable to therapeutic manipulation.

On the basis of previous work showing that release of the neurotransmitter acetylcholine by the vagus nerve can inhibit release of TNF during endotoxaemia, the authors showed that acetylcholine also inhibits endotoxin-induced HMGB1 accumulation in macrophage cultures in a dose-dependent manner. Using specific antagonists of muscarinic ( $\mathrm{mAChR}$ ) and nicotinic (nAChR) acetylcholine receptors, they showed that this effect is due to binding of acetylcholine to nAChR. Indeed, nicotine was more effective than acetylcholine at inhibiting HMGB1 release. The use of antisense oligonucleotides directed against the $\alpha 7$-subunit of $\mathrm{nAChR}$ narrowed down the 'nicotinic anti-inflammatory' pathway to signalling through $\alpha 7 \mathrm{nAChR}$

To establish the mechanism of this antiinflammatory pathway, the authors next showed that nicotine does not induce degradation of HMGB1, does not affect the level of mRNA encoding HMGB1 and does not affect total levels of HMGB1 protein. Instead, nicotine inhibits the endotoxin-induced translocation of HMGB1 from the nucleus to the cytoplasm and thereby inhibits its extracellular secretion. The mitogen-activated protein kinase (MAPK) and nuclear factor- $\mathrm{\kappa B}$ (NF- $\mathrm{\kappa B}$ ) pathways are known to be crucial for macrophage activation and cytokine release; nicotine did not affect endotoxininduced activation of the MAPK pathway but did inhibit $\mathrm{NF}-\kappa \mathrm{B}$ transcriptional activity. However, as the proteinsynthesis inhibitor cycloheximide did not block HMGB1 release by macrophages and as nicotine is thought to inhibit release rather than synthesis of HMGB1, NF- $\kappa B$ probably regulates HMGB1 levels through a non-transcriptional mechanism.

Importantly, the delayed administration of nicotine 24 hours after the induction of sepsis by caecal ligation and puncture - which is a clinically relevant model of human sepsis caused by polymicrobial infection reduced circulating levels of HMGB1, attenuated clinical manifestations of sepsis and significantly improved long-term survival. Therefore, the therapeutic window for the treatment of established human disease with nicotine or other more-specific $\alpha 7 \mathrm{nAChR}$ agonists is far greater than that for neutralization of TNF. Furthermore, as this pathway inhibits the release of HMGB1, it is likely to have fewer side effects in clinical trials than antibody-blocking strategies directed at extracellular HMGB1. Finally, the authors suggest that, because a wide variety of cytokines and mediators, not just endotoxin, converge on activation of the NF- $\mathrm{\kappa B}$ pathway, the nicotinic anti-inflammatory pathway might inhibit HMGB1 release in a range of inflammatory scenarios.

Kirsty Minton

\section{(2) References and links}

ORIGINAL RESEARCH PAPER Wang, $\mathrm{H}$. et al. Cholinergic agonists inhibit

HMGB1 release and improve survival in experimental sepsis. Nature Med.

10, 1216-1221 (2004).

FURTHER READING Tracey, K. J. The inflammatory reflex. Nature 420, 853-859 (2002) 